\title{
Protecting asplenic individuals from fulminant pneumococcal disease
}

H Nohynek (Hanna.Nohynek@thi.fi)1

1. National Institute for Health and Welfare, Helsinki, Finland

Citation style for this article:

Citation style for this article: Nohynek H. Protecting asplenic individuals from fulminant pneumococcal disease. Euro Surveill. 2010;15(23):pii=19584. Available online: http://www.eurosurveillance.org/ViewArticle.aspx?Articleld=19584

In this issue of Eurosurveillance, Chironna et al. report on an unfortunate death of an asplenic individual with fulminant pneumococcal sepsis [1]. Whether or not the death of this particular individual could have been avoided with active preventive interventions remains unanswered. It does, however, raise the alert to revisit the preventive guidelines of asplenic patients in general, and need for reminders both among practicing clinicians as well as asplenic patients.

Asplenia usually results from splenectomy, which is carried out for three main reasons: (i) rupture of the spleen, either because of trauma, during an operation or spontaneous, (ii) as a desired consequence of treatment of certain haematologic disorders, or (iii) because of treatment of neoplasm of the spleen or other organs close to it. Asplenic individuals are at increased risk of fulminant sepsis caused by encapsulated bacteria, especially by Streptococcus pneumoniae, but also by $H$. influenzae and N. meningitidis. Clinicians should consider recommending that asplenic individuals get vaccinated against the latter two as well. Depending on the underlying cause of asplenia, and the baseline incidence of invasive pneumococcal disease (IPD) in a given country, the risk of IPD in asplenic individuals can be as high as 25 times that of the general population [2]. Approximately half of all episodes of overwhelming post-splenectomy infection (OPSI) occur more than five years after splenectomy [3], and case-fatality rates are $50 \%$ or higher, as also demonstrated in the case report by Chironna et al. [1].

What is the best means to protect asplenic individuals from fulminant pneumococcal disease? In Europe, the presently available 23-valent polysaccharide pneumococcal vaccine (23PPV) has been recommended at fiveyear intervals or even more often to asplenic patients [4]. Evidence arising from sufficiently powered randomised controlled trials on the impact of 23PPV in the prevention of OPSI is not available. Concern has been raised about the potential induction of hyporesponse after multiple doses of $23 \mathrm{PPV}$, also after priming with pneumococcal conjugate vaccine [5-6]. The immunological mechanisms behind the hyporesponse to subsequent doses of 23PPV and the clinical relevance of this observation is not fully understood. Immunogenicity studies suggest, however, that certain asplenic individuals might gain protection against IPD even after multiple doses of 23PPV given at five-year intervals [7]. An observational, population-based study carried out in a cohort of asplenic individuals from Denmark is in line with this finding [8], and thus give support to the present clinical practices. On the other hand, there is a subgroup of splenectomised patients with underlying haematologic diseases who clearly do not benefit from 23 PPV and who should be identified by measurement of pneumococcal antibodies after vaccination [9] in order to be provided with other means of protection, such as prophylactic or early antimicrobial treatment.

There is a clear need for the development of more broadly acting, protein-based pneumococcal vaccines, given (i) the limitations of 23PPV in the prevention of pneumococcal disease in several medical risk groups as summarised in a recent meta-analysis published by the World Health Organization [10], (ii) the lack of improved immunity provided by conjugated vaccines to risk groups [11] as well as (iii) the suboptimal coverage of the disease causing pneumococcal serotypes of the presently available pneumococcal conjugate vaccines in these groups combined with the observation of replacement of vaccine serotypes by nonvaccine serotypes [12] especially in risk groups. Proof of clinical efficacy of these new vaccines will be needed, not only in healthy individuals, but also in predefined, immunocompromised risk groups who are most in need of pneumococcal vaccination.

While it will take several years before such new vaccines are licensed, clinicians need to guide and protect their asplenic patients according to the best available knowledge. At this moment the combination of $23 \mathrm{PPV}$, measurement of antibody concentrations induced by 23 PPV and early antimicrobial therapy for those whose protective levels remain low, are the best ways to prevent unnecessary deaths among splenectomised individuals. 


\section{References}

1. Chironna M, Sallustio A, De Robertis A, Quarto M, Germinario C. Fulminant pneumococcal sepsis in an unvaccinated asplenic patient in Italy. Eurosurveill. 2010;15(23): pii 19585. Available from: http://www.eurosurveillance.org/ViewArticle. aspx?Articleld $=19585$

2. Aavitsland P, Froholm LO, Hoiby EA, Lystad A. Risk of pneumococcal disease in individuals without a spleen. Lancet. 1994;344(8935):1504.

3. Waghorn DJ. Overwhelming infection in asplenic patients: current best practice preventive measures are not being followed. J Clin Pathol. 2001;54(3):214-8.

4. Pebody RG, Leino T, Nohynek H, Hellenbrand W, Salmaso S, Ruutu P. Pneumococcal vaccination policy in Europe. Euro Surveill 2005;10(9): pii=564. Available from: http://www. eurosurveillance.org/ViewArticle. aspx?Articleld $=564$

5. Musher DM, Rueda AM, Nahm MH, Graviss EA, RodriguezBarradas MC. Initial and subsequent response to pneumococcal polysaccharide and protein-conjugate vaccines administered sequentially to adults who have recovered from pneumococcal pneumonia. J Infect Dis. 2008;198(7):1019-27.

6. O`Brien KL, Hochman M, Goldblatt D. Combined schedules of pneumococcal conjugate and polysaccharide vaccines: is hyporesponsiveness an issue? Lancet Inf Dis. 2007;7(9):597-606

7. Landgren $\mathrm{O}$, Björkholm $M$, Konradsen HB, Söderqvist $M$, Nilsson B, Gustavsson A et al. A prospective study on antibody response to repeated vaccinations with pneumococcal capsular polysaccharide in splenectomized individuals with special reference to Hodgkin's lymphoma. I Intern Med. 2004;255(6):664-73

8. Ejstrud P, Kristensen B, Hansen JB, Madsen KM, Schønheyder HC, Sørensen HT . Risk and patterns of bacteremia after splenectomy: a population based study. Scand J Infect Dis. 2000;32(5):521-5.

9. Cherif $\mathrm{H}$, Landgren $\mathrm{O}$, Konradsen HB, Kalin M, Björkholm M Poor antibody response to pneumococcal polysaccharide vaccination suggests increased susceptibility to pneumococcal infection in splenectomised patients with haematological diseases. Vaccine. 2006;24(1):75-81.

10. 23-valent pneumococcal polysaccharide vaccine. WHO position paper. Wkly Epidemiol Rec. 2008;83(42):373-84. [Article in English, French] [No authors listed].

11. Goldblatt D, Southern J, Andrews N, Ashton L, Burbidge $P$, Woodgate $S$, et al. The immunogenicity of 7 -valent pneumococcal conjugate vaccine versus 23 -valent polysaccharide vaccine in adults aged $50-80$ years. Clin Infect Dis. 2009;49(9):1318-25.

12. Dagan R. Serotype replacement in perspective. Vaccine. 2009; 27 Suppl 3:C22-4 